\title{
A Young Male with Quadriparesis and Skin Lesions
}

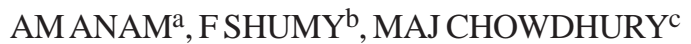

(J Banagladesh Coll Phys Surg 2015; 33: 181-183)

A 21-year-old male presented with spastic quadriparesis, developed gradually over months. He had no pain or restricted movement of neck. Other than features of upper-motor-neuron-lesion of all four limbs, physical examination revealed, multiple Caféau-lait macules ( $>2 \mathrm{~cm}$ in diameter) [Fig. 1], bilateral

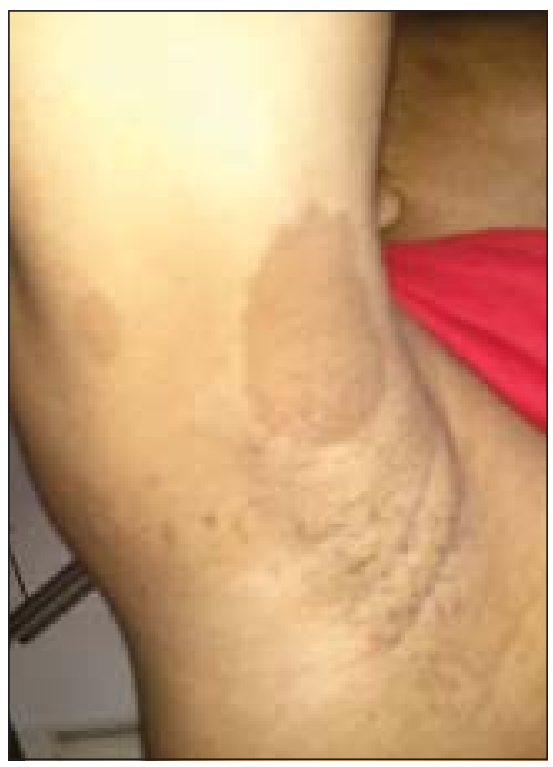

Fig.-1: Café-au-lait macule

a. Dr. Ahmad Mursel Anam, Chief Resident, ICU, Square Hospitals Ltd., 18/F, BU QaziNuruzzamanSarak, Dhaka 1205, Bangladesh.

b. Dr. Farzana Shumy, Medical Officer, Department of Internal Medicine, BangabandhuSheikh Mujib Medical University. Shahbagh, Dhaka-1000, Bangladesh.

c. Prof. M A Jalil Chowdhury, Professor and Chairman, Department of Internal Medicine, BangabandhuSheikh Mujib Medical University. Shahbagh, Dhaka-1000, Bangladesh.

Address of Correspondence: Dr. Ahmad Mursel Anam, Chief Resident, ICU, Square Hospitals Ltd., 18/F, BU Qazi Nuruzzaman Sarak, Dhaka 1205, Bangladesh. Cell: +880-1911-010841, Email: murselanam@gmail.com axillary freckles [Fig. 2], multiple subcutaneous neurofibroma [Fig. 3] scoliosis, and a tender soft tissue mass at right lower chest. Chest $\mathrm{x}$-ray postero-anterior view showed scoliosis, extrathoracic soft tissue shadow with invasion of the pleural space, consistent with plexiform neurofibroma, rib notching and "twistedribbon" ribs[Fig. 4]. Based on clinical and radiological findings, he was diagnosed as neurofibromatosis type 1. MRI of spine revealed multiple neurofibromas, at cervical and dorsal levels. He was offered neurosurgical management. But he decided for treatment at local health-facility, and was lost to follow-up.

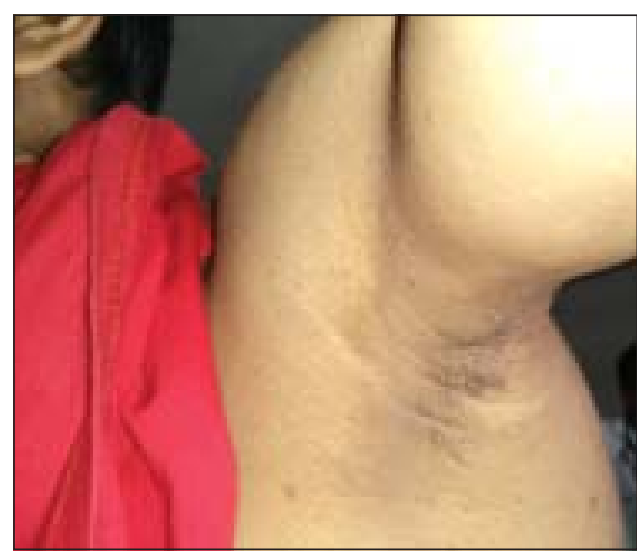

Fig.-2: Crowe's sign / Axillary freckles

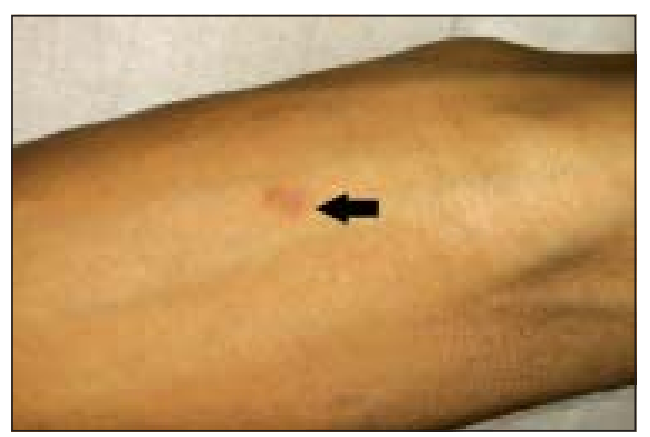

Fig.-3: Subcutaneous neurofibroma 


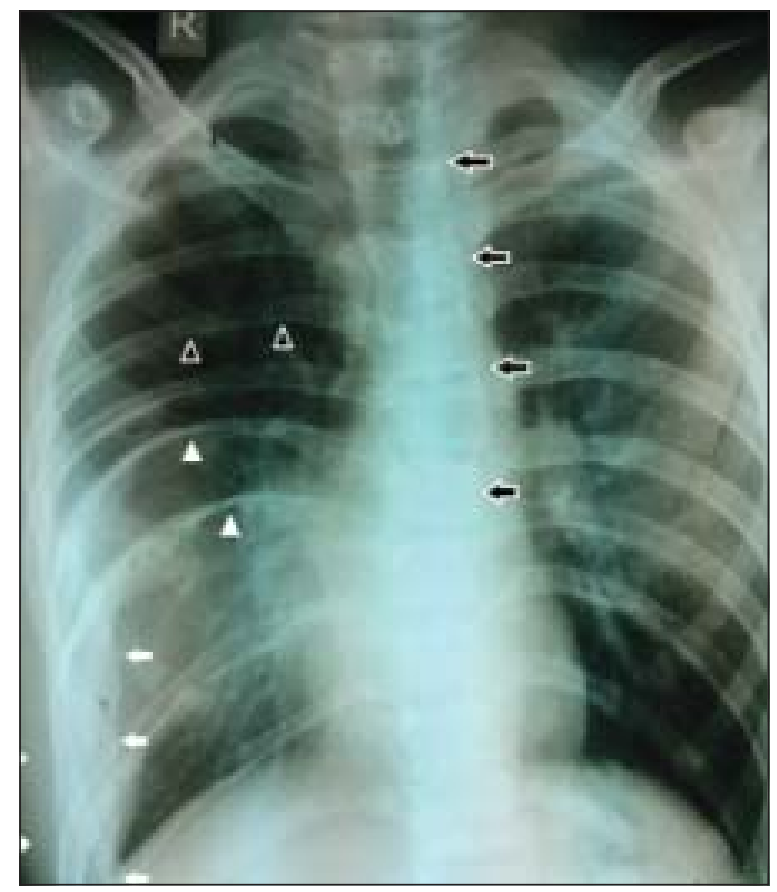

Fig.-4:Chest $x$-ray postero-anterior view showing scoliosis (black arrows), extrathoracic soft tissue shadow with invasion of the pleural space (white arrows), consistent with plexiform neurofibroma, rib notching (black arrow-heads) and "twisted-ribbon" ribs (white arrow-heads)

\section{Discussion:}

von Recklinghausen's disease or Neurofibromatosis type 1, is an autosomal dominant disorder, first described in 1882 by Frederichvon Recklinghausen.It is a relatively common inherited condition (worldwide incidence of $\sim 1$ per 2500 to 3000 individuals, irrespective of age, gender or ethnicity), caused by a germ-line-inactivating mutation in the NF1 gene on chromosome 17q11.2, having high predisposition to develop benign and malignant tumours, and associated with increased mortality and morbidity. The disease manifestation is usually apparent from birth, with varying features of multi-organ involvement, especially the nervous system. ${ }^{1,2}$

Common non-malignant features are café-au-lait macules(earliest clinical manifestation, develops within first 2 years), intertriginous frecklesor Crowe's sign(small, usually found in axillary and inguinal region, but may also be present in areaswhere skinfolds are in apposition, including the neck, above the eyelids and under the breasts in women), Lisch nodules (benignmelanocytic hamartomas ofthe iris that is pathognomonic for NF1,do not impair vision or causeany medical problems,best detected on slit-lamp examination), neurofibromas(benign Schwann-cell tumours, with four subtypes: cutaneous, subcutaneous,nodular or diffuse plexiform, and spinal, result in discomfort or disfigurement, sometimes both sensory and motor deficits), plexiform neurofibromas(arise from multiple nerve fascicles, typically manifest at birth, cancontinue to grow during adolescence and early adulthood, can extend into surrounding structures,causing substantial pain and bone destruction, have a lifetime risk of malignanttransformation), skeletal dysplasia (short stature, osteopenia, scoliosis, sphenoidwing dysplasia, congenital tibial dysplasia, pseudarthrosis), cardiovascular abnormalities, ranging from congenitalheart disease(cardio vascularanomaly, pulmonary artery stenosis) to vasculopathy(renal and cerebral artery stenosis, aortic coarctation,arteriovenous malformations)and hypertension, and neurocognitive deficits. ${ }^{1,2}$ Associated malignant tumors with varying lifetime risk include glioma of the optic pathway, malignant peripheral nerve-sheath tumour, gastrointestinal stromal tumour, breast cancer, leukaemia, phaeochromocytoma, duodenal carcinoid tumour, rhabdomyosarcoma etc. ${ }^{1}$

Diagnosis of neurofibromatosistype 1 is most commonly made using established clinical criteria (National Institute of Health consensus criteria). ${ }^{1,2}$ Two or more of the following clinical features are sufficient toestablish a diagnosis of neurofibromatosis type 1: (1) six or more café-au-lait macules $(>0.5 \mathrm{~cm}$ at largestdiameter in a prepubertal child or $>1.5 \mathrm{~cm}$ in post-pubertalindividuals), (2)axillary freckling or freckling in inguinal regions, (3)two or more neurofibromas of any type or one or moreplexiform neurofibromas, (4)two or more Lisch nodules, (5) a distinctive osseous lesion, (6)an optic pathway glioma, and (7) A first-degree relative with neurofibromatosis type 1diagnosed by the above criteria. ${ }^{3}$ NF1 genetic testing is reserved for unusualpresentations or reproductive decisionmaking. ${ }^{1}$

Routine and cheap investigations like chest radiograph can be very important in diagnosis. Kyphoscoliosis, a hallmark of neurofibromatosis, can easily be visualized, 
if not evident physically. ${ }^{4}$ In chest radiographs, ribs appear notched, due to erosion by neurofibromas in intercostals nerves in the neurovascular grooves, ${ }^{4}$ whereas "twisted-ribbon" ribs reflect mesodermal bone dysplasia. ${ }^{5}$ Plexiform neurofibromas are poorly delineated diffusely infiltratingmultiple masses that arise along the axis of the major nerves, better viewed with CT or MRI. ${ }^{5}$

A multidisciplinary approach to care throughout the lifetime of the patient has been suggested for the appropriate management of neurofibromatosis type 1 . With the enhancing knowledge on the disorder, swift implementation of newer effective treatments is also becomingpossible. ${ }^{1}$ Prompt diagnosis is important to provide optimum care.But because of the varying clinical presentation, patients can present to different medical and surgical specialists and,symptoms might not be recognized. So clinicians must beaware of the diverse features of this disorder, both clinical and radiological, and be vigilant to detect the disorder early.

\section{References:}

1. HirbeAC,GutmannDH. Neurofibromatosis type 1: a multidisciplinary approach to care. Lancet Neurol2014;13(8):834-843.

2. Williams VC, Lucas J, Babcock MA, Gutmann DH, Korf B, Maria BL.Neurofibromatosis type 1 revisited.Pediatrics 2009;123(1):124-133.

3. National Institutes of Health Consensus Development ConferenceStatement: neurofibromatosis. Bethesda, MD, USA, July 13-15, 1987.Neurofibromatosis 1988;1(3):172-178.

4. Muniz MP, Souza AS, Criado DAB, FerrazFilho JRL, Brandão RM, Cardoso LV, Bertollo EMG. Type 1 Neurofibromatosis: Radiological Findings of the Chest. Radiol Bras 2010;43(3):167-170.

5. Khan N,van de Werke I, Ismail F.Neurofibromatosis Revisited: A Pictorial Review. SouthAfrican Journal of Radiology 2010;14(1):16-18. 